\title{
Diacronie
}

Studi di Storia Contemporanea

$N^{\circ} 7,3 \mid 2011$

«Spagna Anno Zero»: la guerra come soluzione

\section{Memorie difficili}

Antifascismo italiano, volontariato internazionale e guerra civile spagnola

\section{Enrico Acciai}

\section{Q OpenEdition \\ Journals}

\section{Edizione digitale}

URL: http://journals.openedition.org/diacronie/3392

DOI: 10.4000/diacronie.3392

ISSN: 2038-0925

Editore

Association culturelle Diacronie

Notizia bibliografica digitale

Enrico Acciai, « Memorie difficili », Diacronie [Online], N 7, 3 | 2011, documento 19, Messo online il 29 juillet 2011, consultato il 20 avril 2019. URL : http://journals.openedition.org/diacronie/3392 ; DOI : 10.4000/diacronie.3392 


\section{Diacronie}

\section{Memorie difficili}

\section{Antifascismo italiano, volontariato internazionale e guerra civile spagnola}

\section{Enrico ACCIAI *}

Sin dalla conclusione della guerra civile l'intervento dell'antifascismo italiano in Spagna ha generato più memorie spesso in conflitto tra di loro. Tra il 1936 ed il 1939 quello italiano alla causa repubblicana fu un contributo collettivo, come non lo fu per nessun altro gruppo nazionale, che coinvolse tutte le famiglie politiche antifasciste. Per cercare di comprendere l'origine di quella che potremmo chiamare una vera e propria "atomizzazione della memoria" si dovranno analizzare le molteplici eredità dell'esperienza spagnola tanto nel difficile biennio 1943-1945 quanto durante i primi anni del dopoguerra.

\section{Introduzione}

I

n quest'articolo si cercheranno di individuare i caratteri, come vedremo congeniti a quell'esperienza, che avrebbero determinato una pluralità di memorie dell'intervento antifascista italiano nella guerra civile spagnola. In un secondo momento, ci sposteremo avanti, sin dopo la conclusione della seconda guerra mondiale, per vedere come lo sviluppo di queste memorie sia stato profondamente legato alle questioni irrisolte del nuovo Stato repubblicano ed, in particolare, al difficile rapporto tra antifascismo e democrazia. Imposteremo quindi l'analisi su due piani: da un lato analizzeremo perché l'intervento italiano non abbia generato, come invece è successo per altri casi nazionali, una memoria unica e dall'altro cercheremo di capire com'è stato possibile che nel secondo dopoguerra ci sia stata una divisione in "forti" e "deboli" di queste memorie. Come si vedrà, questi due piani si rivelano entrambi imprescindibili per provare a capire tanto perché persista ancora 
oggi una memoria "confusa" dell'intervento italiano nella guerra civile spagnola, quanto il motivo del ritardo con cui la storiografia italiana si è dedicata a questo tema.

Secondo Jaques Delperrié de Bayac, l'Italia è stato il paese dove il mito delle Brigate Internazionali si è conservato e si è tramandato con più forza. ${ }^{1}$ Un'affermazione sicuramente vera, sono infatti pochi i paesi dove sia ancora viva e attiva un'associazione omologa all'AICVAS [Associazione Italiana Combattenti Volontari Antifascisti di Spagna, N.d.A.] o dove un evento dal quale sono ormai passati più di settant'anni sia ancora in grado di sollevare l'interesse dell'opinione pubblica, ma dietro la quale si nasconde il percorso complicato e tutt'altro che lineare che ha conosciuto la memoria di quel volontariato, un percorso che in queste pagine cercheremo di ripercorrere.

\section{Radici per un pluralismo di memorie}

I presupposti per una pluralità di memorie erano connaturati allo stesso intervento in Spagna: tra l'estate del 1936 (quando cominciarono ad arrivare i primi volontari italiani) ed i primi mesi del 1939 (quando i reduci delle Brigate Internazionali abbandonarono una Spagna repubblicana ormai collassata) si andarono infatti delineando quelle memorie che sarebbero sopravvissute per decenni e che, in parte, insistono ancora oggi. Come si è detto, l'origine di questo processo si deve individuare nell'estate del 1936; quello italiano in Spagna, rispetto ad altri casi nazionali, fu infatti un contributo particolare. Se, ad esempio, per quanto riguarda i francesi o gli inglesi il gruppo politico che espresse la maggior parte dei volontari tese a coincidere con quello dei militanti o dei simpatizzanti comunisti, tra gli italiani si registrò invece una "pluralità" di famiglie politiche coinvolte. Non essendo questa la sede per indagare le origini di queste differenze nazionali, basti ricordare la peculiarità italiana: con una dittatura che a metà anni Trenta aveva ormai festeggiato il proprio decennale e con un variegato movimento antifascista che non esisteva in altri casi europei gli italiani si candidarono, sin dall'inizio, a giocare un ruolo del tutto specifico nelle vicende iberiche.

Lo scoppio delle ostilità in Spagna colse l'antifascismo italiano in un momento particolare della propria storia: la svolta seguita al VII congresso del Comintern e l'avvento della stagione dei Fronti Popolari avevano modificato i rapporti tra gli esuli italiani. I comunisti, in particolare, erano passati nel volgere di pochi mesi dal "social fascismo" degli anni precedenti ad un dialogo con le altre forze antifasciste, tanto che nell'estate del 1934 si era arrivati alla stipula di un patto d'unità tra PCd'I e PSI. Anche

${ }^{1}$ SKOUTELSKY, Remi, Novedad en el frente: Las Brigadas Internacinonales en la guerra civil, Madrid, Temas de Hoy, 2006, p. 444. 
la nascita di GL, fondata nel 1929 subito dopo la rocambolesca fuga di Carlo Rosselli dalla colonia di confino di Lipari, aveva portato un'aria nuova nel mondo dell'esilio antifascista: quest'ultimo aveva sin da subito lavorato per trovare un elemento comune tra tutte la «migliori energie» di quell'universo così variegato ${ }^{2}$. Perfino gli anarchici, tradizionalmente i più restii a collaborazioni con altre forze politiche, avevano accolto con interesse la nascita di questo nuovo movimento. Luigi Fabbri, uno dei grandi vecchi del movimento libertario italiano, aveva definito l'evento come un "fatto rivoluzionario" e aveva scritto che GL avrebbe potuto essere «un fattore di primo ordine per la rivoluzione italiana»3. In sintesi, si potrebbe dire che all'interno dell'antifascismo in esilio (l'ambiente dal quale sarebbe provenuta la maggior parte dei volontari) nei primi anni trenta si può segnalare, quanto meno, un serio dialogo tra le diverse famiglie politiche. Un dato del genere emerge se si analizza la socialità degli esuli italiani. In questo periodo le relazioni dei confidenti fascisti rilevarono spesso la compresenza di militanti di diverse tendenze politiche alle iniziative più disparate: nel novembre del 1935, ad esempio, ad una riunione settimanale di GL vennero segnalati «diversi anarchici, repubblicani e massimalisti», mentre il mese successivo ad una conferenza pubblica molto partecipata, anch'essa giellista, sarebbero stati presenti molti anarchici che avrebbero animato «con entusiasmo» il dibattito. 4

Nel giugno del 1936, poche settimane prima dello scoppio della guerra civile spagnola, Carlo Rosselli ribadì per l'ennesima volta la propria volontà di perseguire una qualche forma di unità tra le diverse famiglie politiche dell'antifascismo: «Reagire con centuplicata energia. Ricercare l'interesse e la capacità d'interesse attraverso interessi fortissimi. Ridestare passioni affettive», scrisse in quell'occasione, «condizione prima del riscatto non è la guerra, il terremoto generale che troppi ancora oggi si augurano. È la capacità dell'antifascismo di farsi esso una cosa sempre più viva e ricca, tale da interessare e appassionare i propri membri innanzitutto e da vivere di vita propria. L'autonomia: riprova di ogni ideale autentico. Quindi meno politica in senso angusto e burocratico e più cultura e umanità, soprattutto in esilio».5

\footnotetext{
2 FEDELE, Santi, Storia della Concentrazione Antifascista, 1927-1934, Milano, Feltrinelli, 1976, pp. 74-75. Per vedere le vicende che portarono alla fondazione del movimento giellista si veda la recente sintesi: GIOVANA, Mario, Giustizia e Libertà in Italia - Storia di una cospirazione antifascista, 1929-1937, Torino, Bollati Boringhieri, 2005, pp. 3-23.

3 Citato in: FEDELE, Santi, Luigi Fabbri, un libertario contro il bolscevismo e il fascismo, Pisa, BFS, 2006, p. 72.

4 Archivio Centrale dello Stato (ACS), Divisione Polizia Politica (DPP), Fondo per Materia (FM) pacco 127, fascicolo 1, sottofascicolo D. Relazione informatore K7/53, 25/11/1935 e ACS, Casellario Politico Centrale (CPC), busta 537, fascicolo 737478 Berneri Camillo. Appunto Direzione Generale PS, 28/12/1935.

5 ROSSELLI, Carlo, Scritti dall'Esilio, Volume II, dallo scioglimento della concentrazione antifascista alla Guerra di Spagna (1934-1937), Torino, Einaudi, 1992, p. 378.
} 
Pur esistendo quindi i presupposti per un intervento unitario dell'antifascismo italiano nella guerra civile, questo si produsse? La risposta è negativa. In Spagna, infatti, i volontari italiani non s'integrarono solamente alle Brigate Internazionali, ed in particolare al celebre battaglione Garibaldi, ma diedero vita almeno ad un'altra esperienza significativa: la Sezione Italiana della Colonna Ascaso ${ }^{6}$. Camillo Berneri e Carlo Rosselli fondarono questo gruppo a cavallo tra il luglio e l'agosto del 1936 a Barcellona; la Sezione avrebbe visto passare tra le sua fila più di 600 antifascisti italiani e si sarebbe sciolta nell'aprile dell'anno successivo. La maggior parte dei suoi integranti, circa il $60 \%$, furono anarchici, mentre un 14\% fu comunista; tanto i socialisti quanto i giellisti si attestarono invece attorno al 6\%; come emerge da questi dati, il gruppo si determinò necessariamente come colonna libertaria. La Sezione Italiana combatté sul fronte aragonese, al lato delle milizie anarco-sindacaliste catalane ed ebbe il proprio battesimo del fuoco il 28 di agosto, quando le posizioni degli italiani furono attaccate dalle truppe nazionaliste; secondo il giornalista giellista Umberto Calosso, presente quel giorno sul fronte, si sarebbe trattato del «primo fatto d'armi partigiano degli italiani nella guerra europea»7. La colonna si sarebbe sciolta nell'aprile dell'anno successivo quando i suoi componenti, nella quasi totalità anarchici (i giellisti ed i socialisti avevano abbandonato il gruppo durante i primi giorni di gennaio), si rifiutarono di accettare la militarizzazione imposta dal governo repubblicano.

Alcuni mesi dopo rispetto al gruppo di Rosselli e di Berneri, tra l'ottobre ed il novembre del 1936, si organizzarono le Brigate Internazionali e al loro interno vide la luce il battaglione (in un secondo momento brigata) Garibaldi ${ }^{8}$. Questa fu l'altra grande esperienza dell'antifascismo italiano in Spagna, un'esperienza che avrebbe avuto una vita più lunga rispetto alla Sezione Italiana e che sarebbe sopravvissuta fino all'autunno del 1938 quando il governo repubblicano decise di ritirare i volontari stranieri. I garibaldini di Spagna furono sicuramente almeno 4.00o (ancora non esiste un dato

\footnotetext{
${ }^{6} \mathrm{Al}$ riguardo mi permetto di segnalare i miei due recenti saggi: ACCIAI, Enrico, «Berneri e Rosselli in Spagna, l'esperienza della "Sezione Italiana della Colonna Ascaso"», Spagna Contemporanea, 2010, n. 38) e ACCIAI, Enrico, «Una scelta di vita. Il primo volontariato italiano nella guerra civile spagnola», Storia e problemi contemporanei, 2010, n. 55).

7 CALOSSO, Umberto, «La battaglia di Monte Pelato», in ROSSI, Ernesto (a cura di), No al fascismo, Torino, Einaudi, 1957, p. 252. Si veda anche la mia tesi di dottorato: ACCIAI, Enrico, Viaggio attraverso l'antifascismo. Volontariato internazionale e guerra civile spagnola: la Sezione Italiana della Colonna Ascaso, Dottorato di Ricerca - XXII Ciclo, Università degli Studi della Tuscia, Viterbo, 2010.

$8 \mathrm{Al}$ riguardo si veda: DELPERRIE DE BAYACA, Jaques, Les Brigades internacionales, Parigi, Fayard, 1968 ed il più recente SKOUTELSKY, Remi, op. cit.
} 
certo ma siamo d'accordo con Gianni Isola che questa stima andrà rivista al rialzo). ${ }^{9}$ In questo caso, secondo quanto emerge dai dati da noi raccolti negli ultimi anni, i comunisti rappresentarono ben il 71\% dei volontari, seguiti da un 10\% di socialisti, da un $4,5 \%$ di anarchici e da uno $0,5 \%$ di giellisti. Per questo secondo gruppo non è quindi sbagliato parlare di una formazione spiccatamente comunista.

Quindi, l'intervento antifascista italiano in Spagna non riuscì ad essere unitario. Nonostante il 29 luglio del 1936 Carlo Rosselli avesse scritto al repubblicano (e futuro comandante del battaglione Garibaldi) Randolfo Pacciardi come «in tutti i movimenti e naturalmente in vivissima forma nel nostro» vi fosse «il desiderio di arrivare possibilmente ad un intervento collettivo seriamente organizzato e selezionato dell'antifascismo italiano», questo appello rimase inascoltato: questo non fu possibile ${ }^{10}$. Il leader giellista fu uno dei più attivi sostenitori della necessità dell'unità antifascista tra i volontari italiani; pochi giorni prima della sua partenza per Barcellona, ad esempio, aveva inviato un ultimo accorato appello a comunisti e socialisti affinché partecipassero alla colonna che stava organizzando con Berneri: «sarebbe bene riunirsi e riunire le forze», scrisse in quell'occasione, «perché il contributo italiano sia il più largo ed efficace possibile» ${ }^{11}$. Ma i comunisti, e sulla loro scia i socialisti, preferendo aspettare il via libera da Mosca, rifiutarono quella proposta; il Comintern avrebbe deciso per un sostegno attivo alla causa repubblicana («tra i lavoratori di tutti i paesi», avrebbe recitato una nota, «si dovrà favorire un arruolamento di volontari da inviare in Spagna che conoscano la disciplina militare») soltanto durante la riunione del Presidium del 16 settembre, quindi un mese e mezzo dopo la partenza di Carlo Rosselli per la Spagna. ${ }^{12}$

Se da un lato l'origine di quello che abbiamo definito un pluralismo di memorie può essere facilmente individuato proprio in questa esistenza di almeno due esperienze che videro coinvolti dei volontari italiani; dall'altro l'evoluzione delle vicende politiche all'interno del bando repubblicano non avrebbe favorito una "facile convivenza" tra queste diverse memorie. I contrasti tra le forze politiche e sindacali spagnole che

\footnotetext{
9 ISOLA, Gianni, «La contribution du Parti communiste d'Italie aux Brigades internationales», in PREZIOSO, Stefanie, BATOU, Jean, RAPIN, Jaques (a cura di), Tant pis si la lutte est cruelle - Volontaires internationaux contre Franco, Parigi, Editions Syllepse, 2008, pp. 78-79.

10 Istituto Storico della Resistenza in Toscana (ISRT), Archivi di Giustizia e Libertà (AGL), Fondo Carlo Rosselli, busta 1, fascicolo 86. Lettera di Carlo Rosselli a Randolfo Pacciardi, 29/07/1936.

${ }^{11}$ Archivio Fondazione Gramsci (AFG), Fondo Partito Comunista d'Italia (FPCdI), fascicolo 1397

- Corrispondenza della dirigenza del PCI con "Giustizia e Libertà", folio 15. Lettera di Carlo Rosselli alla dirigenza del PCd’I e del PSI, 01/o8/1936.

${ }^{12}$ SCHAUFF, Frank, La victoria frustrada: la Unión Soviética, la Internacional Comunista y la guerra civil española, Barcellona, Debate, 2008, p. 178.
} 
portarono alle celebri giornate barcellonesi del maggio 1937 ed, in pratica, alla repressione dell'anima più radicale del movimento libertario iberico ebbero dei riflessi anche tra gli italiani ${ }^{13}$. Le prime crepe emersero nel gennaio del 1937 quando Carlo Rosselli ed i suoi uomini abbandonarono la Sezione Italiana perché in aperto disaccordo con la maggioranza anarchica; nell'aprile di quell'anno Aldo Garosci avrebbe riferito ad alcuni militanti giellisti come gli anarchici, con le loro prepotenze, avessero ormai «screditato il nome degli italiani»14. Ma in realtà la frattura più grande si produsse tra libertari e comunisti sulla scorta delle vicende del maggio 1937: durante quelle giornate molti degli ormai ex-componenti della Sezione Italiana si trovarono dietro le barricate al lato di coloro che ne sarebbero usciti sconfitti. Camillo Berneri venne addirittura assassinato a sangue freddo, in compagnia del compagno Francesco Barbieri, da un gruppo di militanti stalinisti del PSUC) ${ }^{15}$. Alcune settimane più tardi, il 29 maggio, sul foglio comunista parigino Il Grido del Popolo venne pubblicato un pezzo nel quale si diceva che il Berneri era stato «giustiziato dalla rivoluzione democratica, a cui nessun antifascista può negare il diritto di legittima difesa» ${ }^{16}$. Fu così che dall'estate del 1937 per alcuni volontari italiani la vita in Catalogna ed in Spagna cominciò a diventare complicata e finirono anche tra le vittime della repressione repubblicana. Tra chi venne addirittura incarcerato ci fu, ad esempio, il livornese Enzo Fantozzi che era stato tra i primi ad arrivare in Spagna ${ }^{17}$. Aldo Aguzzi, collaboratore di Berneri, avrebbe ben sintetizzato cosa dovettero provare i suoi compagni d'armi a partire dal maggio 1937: quei fatti, scrisse pochi mesi dopo, «avevano approfondito il dissenso, gli anarchici italiani si sentivano sempre più allontanati dalla tattica dei compagni spagnoli. Finite quelle tragiche giornate, essi si sentivano ancor più profondamente disillusi nelle loro speranze» ${ }^{18}$. Com'è naturale che fosse, queste vicende avrebbero avuto un peso importante sulle memorie "generate" dai volontari: i libertari non

13 Al riguardo si vedano i due recenti lavori: GALLEGO, Ferran, Barcelona, mayo 1937, Barcellona, Debate, 2007 e VIÑAS, Angel, El escudo de la República - El oro de España, la apuesta sovietica y los hechos de mayo de 1937, Barcellona, Critica, 2007.

${ }_{14}$ ACS, DPP, FM pacco 127, fascicolo 1 sottofascicolo E. Relazione informatore $\mathrm{K}_{7} / 53$, 29/04/1937.

15 Il grosso dei reduci della Sezione Italiana (circa 200 uomini), quando a Barcellona scoppiarono i violenti scontri, era riunito nella caserma Spartacus e qui si organizzò per difendersi sotto il comando del bolognese Gelindo Zanasi (AGUZZI, Aldo, «I fatti del maggio», in Un trentennio di attività anarchica (1914-1945), Cesena, Edizioni Antifastato, 1953, p. 194). Sulla morte di Berneri si veda, tra le tante testimonianze: PATRICK, Jeane, "Quatros cartas desde Barcelona», in Barcelona, mayo 1937 - Testimonios desde las barricadas, Barcellona, Alikornio, 2006, p. 170.

${ }^{16}$ Citato in: DE MARIA, Carlo, Camillo Berneri - Tra anarchismo e liberalismo, Milano, Franco Angeli, 2004, p. 111.

${ }_{17}$ ACS, CPC busta 1954, fascicolo 19862 Fantozzi Enzo. Appunto della Divisione Affari Generali e Riservati, 26/04/1937.

18 AGUZZI, Aldo, op. cit. 
avrebbero mai perdonato ai comunisti, anche a quelli italiani, per il "trattamento" loro riservato in Spagna.

Le tensioni e le frizioni tra le diverse famiglie politiche non risparmiarono il battaglione Garibaldi. Nonostante in una prima fase, almeno fino all'estate del 1937, fosse sembrato possibile salvaguardare una pacifica convivenza tra gli antifascisti italiani, con l'allontanamento del repubblicano Randolfo Pacciardi dal comando dei garibaldini, quella che era un'egemonia numerica dei comunisti si trasformò, di fatto, in un controllo politico sulla totalità del battaglione. In quelle stesse settimane, sul piano politico spagnolo, ci fu il passaggio dal testimone di presidente dal consiglio da Largo Cabellero a Juan Negrín, considerato più vicino alle posizioni comuniste rispetto al suo predecessore, e si produsse la dura repressione dell'ala più radicale del movimento libertario ${ }^{19}$. La leader comunista Teresa Noce, in un rapporto redatto ad inizio 1938, affermò come il lavoro di Partito "propriamente detto» fosse cominciato proprio «dal mese di giugno 1937», quindi in concomitanza con l'allontanamento del Pacciardi ${ }^{20}$. Nella vicenda entrò anche Pietro Nenni, con un rapporto alla direzione del PSI nel quale accusava i comunisti di essersi comportati scorrettamente e di aver tradito il patto unitario fondante dell'esperienza spagnola. ${ }^{21}$

Sempre nell'estate del 1937, la sostituzione nel ruolo di commissario politico di Antonio Roasio con Pietro Pavanin, considerato più vicino alla linea ufficiale del partito, fu un altro chiaro indizio nella direzione di un rinnovato protagonismo comunista a scapito delle altre forze politiche ${ }^{22}$. Secondo Teresa Noce, a partire da quelle settimane sarebbero stati nominati dei quadri comunisti fidati in ruoli chiave a Madrid, ad Albacete, a Valencia e sul fronte: «questi compagni», aggiunse la Noce, «si misero subito al lavoro per organizzare il partito nella brigata e nelle località dove si trovavano. Venne fatto un censimento dei compagni comunisti italiani e si cominciò il lavoro di passaggio al PCS, sotto il controllo della compagna Estella, del compagno Pavanin per i quadri di Albacete e del compagno Giordano che lavorava presso il CC del partito spagnuolo» ${ }^{23}$. Nell'ottobre del 1938, al momento del ritiro delle BI, su 20 commissari politici della Garibaldi ben 19 sarebbero stati comunisti e solo uno

\footnotetext{
19 VIÑAS, Angel, op. cit., pp. 549-574.

${ }^{20}$ Centro Russo per la Conservazione degli Archivi di Storia Politica e Sociale (RGASPI), Fondi del Comintern (FC), fascicolo 545-3-153, folio 109. Rapporto sul lavoro svolto dai comunisti italiani nelle brigate internazionali (giugno-dicembre 1937), sd.

${ }^{21}$ RGASPI, FC, fascicolo 545-1-027, folio 100-102. Rapporto del segretario alla direzione, 08/09/1937.

${ }^{22}$ RGASPI, FC, fascicolo 545-6-002, folio s/n. Lettera di D’Onofrio a Pavanin, 18/o7/1937.

23 Ibidem.
} 
socialista ${ }^{24}$. Come ha giustamente scritto qualche anno fa Gabriele Ranzato, «c'è un'inerzia fronte populista nel continuare a dire che nelle Brigate Internazionali si batterono fianco a fianco comunisti, anarchici, socialisti, repubblicani»; in realtà, e come abbiamo visto in particolar modo dopo l'estate del 1937, l'egemonia comunista fu netta ed incontestabile. ${ }^{25}$

Nelle ultime pagine si è visto quanto, alla prova dei fatti, si fosse rivelato frammentato l'intervento antifascista in Spagna. I presupposti per quelle che si sarebbero sviluppate come delle memorie divise erano quindi già presenti durante la guerra civile. Furono in pochi a cogliere questa peculiarità sin da subito, tra di loro crediamo che valga la pena ricordare il dirigente repubblicano Fernando Schiavetti. Già nell'aprile del 1937, in una circolare indirizzata ai quadri del proprio partito, lo Schiavetti colse i limiti e le aporie dei diversi antifascismo in Spagna: «approfittando del prestigio di cui la Russia e la III Internazionale sono venute a godere per la loro azione in Spagna, i comunisti italiani proseguono alacremente la realizzazione delle ultime e generali direttive (fronte unico al di sopra delle classi, "riconciliazione", difesa della democrazia, ecc.) dinnanzi alle quali gli altri partiti sono stati per un po’ di tempo ostili e diffidenti. Sotto l'impulso di questa energica iniziativa, condotta con straordinaria abbondanza di mezzi, una parte dell'antifascismo (elementi socialisti, repubblicani, GL) sembra piegarsi, polverizzarsi, perdere la propria personalità per riunirsi in una massa amorfa e disorientata, abilmente utilizzata dallo stato maggiore comunista per il raggiungimento dei fini indicati da Mosca; un'altra parte invece (massimalisti, anarchici, comunisti dissidenti) s'irrigidisce in un'opposizione acre e settaria, priva di possibilità costruttiva, che impedisce di apprezzare quel che v'è di giusto e di fecondo nell'attività comunista» ${ }^{26}$. Poche efficaci righe che, come vedremo, sono utili per comprendere quanto sarebbe successo negli anni successivi.

\footnotetext{
24 RGASPI, FC, fascicolo 545-1-061, folio s/n. Elenco dei commissari politici delle Brigate Internazionali, 10/1938.

25 RANZATO, Gabriele, «Ripensare la guerra di Spagna», in: COLLOTTI, Enzo (a cura di), Fascismo e antifascismo - Rimozioni, revisionismi, negazioni, Roma-Bari, Laterza, 2000, p. 143.

${ }^{26}$ DE MARIA, Carlo, op. cit., p. 111.
} 


\section{Il dopoguerra e la difficile memoria}

Ancora oggi, nel dover mettere in relazione il volontariato internazionale e la guerra civile spagnola, saremmo sicuramente indotti a pensare al battaglione Garibaldi ed al movimento comunista; altrettanto rapidamente potrebbe poi nascere un'associazione tra quest'esperienza e quella della lotta partigiana in Italia durante il biennio 19431945. Simili relazioni, per quanto possano sembrare naturali, sono in realtà il frutto di una lunga sedimentazione delle memorie; una sedimentazione che è cominciata, sulla scorta di quanto si è detto nelle pagine precedenti, nell'immediato dopoguerra e che ha generato un cortocircuito che è andato ben oltre la mera ricerca storica. In realtà, nell'Italia del dopoguerra, le memorie di chi aveva combattuto in Spagna seguirono vicende diverse e, spesso, alternative; nella seconda parte di questo saggio si vedrà come se quella dei battaglione Garibaldi (in particolare dei volontari comunisti e socialisti) si sarebbe andata determinando come "memoria forte", dall'altro lato ai militanti libertari ed, in parte, ai giellisti sarebbe rimasta una "memoria debole". Questo processo fu, come si è detto in apertura, strettamente legato alle vicende politiche dell'immediato dopoguerra, e da li partiremo.

Lo scoppio della guerra civile in Spagna aveva segnato l'inizio del decennio più violento della storia europea e solo con la conclusione della seconda guerra mondiale nella primavera del 1945 si sarebbe definitivamente usciti da quella che è stata giustamente definita una guerra civile continentale durata più di trent'anni. ${ }^{27} \mathrm{Il}$ crollo del regime nazista rappresentò, a livello simbolico, una netta cesura con il passato recente: per i sopravvissuti ai drammi collettivi che avevano sconvolto le società ci sarebbe stato, rispetto a quel momento, un prima ed un dopo. C'era chi, come gli italiani, i tedeschi, gli austriaci o gli ungheresi, aveva vissuto per decenni sotto dei regimi dittatoriali, o chi, questo era il caso delle generazioni più anziane, era dovuto passare attraverso due guerre mondiali; c'erano poi le decine di migliaia di uomini e di donne che avevano conosciuto l'esperienza dell'esilio; i milioni che avevano subito la violenza di una guerra totale e, soprattutto, c'erano i sopravvissuti dei genocidi scatenati dalla follia nazifascista. La seconda guerra mondiale fu un trauma condiviso da tutto un continente: la percentuale delle vittime civili non era mai stata così alta in

27 Cfr. TRAVERSO, Enzo, A ferro e fuoco - La guerra civile europea, 1914-1945, Bologna,Il Mulino, 2007. Tra i primi a parlare di guerra civile europea ci fu, già dagli anni trenta, il giurista tedesco Carl Schmitt (SCHMITT, Carl, Le categorie del politico: saggi di teoria politica, Bologna, Il Mulino, 1972), il concetto fu poi ripreso con notevole successo, e con molte polemiche, da Ernst Nolte (NOLTE, Ernst, Nazionalismo e bolscevismo: la guerra civile europea, 1917-1945, Milano, Sansoni, 1988). 
alcun conflitto precedente e l'immane livello di sofferenze e devastazioni patito per più di sei anni avrebbe profondamente segnato le società europee durante gli anni a venire $^{28}$. «Alla fine della seconda guerra mondiale», ha scritto Tony Judt, «l'Europa offriva uno spettacolo di miseria e desolazione totali. Le foto e i documenti del tempo mostrano penose fiumane di civili disperati che arrancano attraverso un desolato paesaggio di città diroccate e campi abbandonati. Bambini rimasti senza genitori si aggirano tristemente in mezzo a gruppi di donne esauste che frugano tra mucchi di macerie. [...] Tutto e tutti (con la sola eccezione delle ben nutrite forze di occupazione alleate) sembrano esausti, privi di ogni risorsa, completamente stremati» ${ }^{29}$.

Era chiaro che non sarebbe stato facile elaborare la memoria, le memorie, degli anni precedenti ${ }^{30}$. Per risanare le ferite che si erano aperte durante il secondo conflitto mondiale fu quindi necessario del tempo; ancora nel 1954 il giurista Piero Calamandrei avrebbe ricordato come, nonostante fossero passati ormai dieci anni dalla fase "resistenziale", non fosse ancora facile afferrarne il significato storico. "Quei fatti sono ancora, per chi li ha vissuti», affermò il Calamandrei davanti ad un platea di giovani studenti, «passione e dolore: le cicatrici dentro di noi dolgono ancora; certe ferite sono ancora aperte; e a rivederci innanzi certe facce, e risentir certi nomi, ci si accorge che il lutto e lo sdegno è ancora immutato ${ }^{3}{ }^{3}$. Le parole di Calamandrei, riferite alla vicende della resistenza italiana, potrebbero essere benissimo estese all'insieme delle società europee di quegli anni; «e poi è risaputo», continuava il giurista, «che ogni giudizio storico, anche di venti che paion lontani nel tempo, è sempre un giudizio sul presente e, insieme, una interrogazione indirizzata all'avvenire. [...] Mai come questa volta è vero che fare la celebrazione del passato vuol dire guardar dentro noi e fare il nostro esame di coscienza»32. È In questo panorama "fluido", in costante bilico tra il ricordo e la rimozione dei traumi appena vissuti, che devono essere collocate le vicende di chi era stato volontario in Spagna; fu nel decennio tra gli anni Quaranta e Cinquanta che le loro memorie si sarebbero andate sviluppando secondo una molteplicità di declinazioni, tanto politiche quanto nazionali.

Concentriamoci sul caso italiano. In Italia il momento simbolico di cesura con il passato fu rappresentato dal 25 aprile; una data che rappresenta, ha scritto Pietro

${ }^{28}$ MAZOWER, Mark, Le ombre dell' Europa: democrazie e totalitarismi nel XX secolo, Milano, Garzanti, 2005, pp. 213-214.

29 JUDT, Tony, Dopoguerra. Com'è cambiata l'Europa dal 1945 a oggi, Milano, Mondadori, 2007, p. 19.

$3^{\circ}$ Cfr. JUDT, Tony, «Preface», in DEAK, Istvan, GROSS, Jan, JUDT, Tony (a cura di), The politics of retribution in Europe - World War II and its Aftermath, Princeton, Princeton University Press, 2000.

${ }^{31}$ CALAMANDERI, Piero, Uomini e città della resistenza, Roma-Bari, Laterza, 1977, p. 3.

32 Ibidem, pp. 3-4. 
Scoppola, «qualcosa di decisivo per la storia del paese: punto d'arrivo di una vicenda drammatica, punto di partenza della ricostruzione della democrazia italiana. Vi sono tutti gli elementi per fare di questa data un elemento di forte riferimento per la storia del popolo italiano»33. Per gli italiani la conclusione del secondo conflitto mondiale sancì l'uscita da una dittatura ventennale e, in alcune zone del Paese, da una cruenta guerra civile. Tornando di poco indietro, l'estate del 1943 aveva rappresentato un ulteriore passaggio chiave in questo processo: lo sbarco degli alleati in Sicilia, l'arresto di Mussolini, l'8 settembre, la fondazione della Repubblica sociale e l'inizio della guerra partigiana si rivelarono dei nodi centrali per capire, sul lungo periodo, la storia italiana successiva ${ }^{34}$. Per quanto riguarda il nostro caso di studio, fu decisivo il passaggio, in quelle settimane, di vasti settori antifascisti alla lotta armata e, soprattutto, la nascita dell'idea che il movimento antifascista, nonostante la sua lunga storia, potesse essere considerato una sorta di appendice di quello resistenziale (antifascismo come "sfera politica della resistenza")35. Inoltre, nei venti mesi che durò, la guerra partigiana, pur coinvolgendo attivamente e passivamente una buona parte della popolazione italiana, non arrivò mai ad essere quell'esperienza che avrebbe potuto fungere da "mito fondante condiviso" per la comunità nazionale del futuro Stato repubblicano e, anzi, sembrò portare con sé tutte le caratteristiche per determinarsi come agente di frattura delle successive vicende. L'Italia del dopoguerra si sarebbe, infatti, scoperta ben presto nuovamente divisa (basti pensare, a livello simbolico, allo scarsissimo scarto con cui, nel luglio del 1946, l'opzione repubblicana si impose su quella monarchica). Quasi vent'anni fa Silvio Lanaro, nelle prime pagine della sua Storia dell'Italia repubblicana, è stato in grado di dipingere egregiamente questo passaggio cruciale nella storia del nostro paese. 36

I partiti che emersero vittoriosi dalla guerra civile, le nuove classi dirigenti, avevano urgenza tanto di legittimarsi per l'imminente futuro democratico quanto di smarcasi dal passato regime e la Resistenza sembrava offrire le caratteristiche ideali per questa doppia necessita. Il processo fu particolarmente interessante a sinistra. In buona parte d'Europa, infatti, le forze politiche di quest'area, ed in particolare i partiti comunisti, assunsero una centralità fino a quel momento inedita: quello italiano, quello francese, quello jugoslavo e quello greco divennero degli attori di primissimo piano dei rispettivi

33 SCOPPOLA, Pietro, 25 aprile: liberazione, Torino, Einaudi, 1995, p. 5.

34 Cfr. PAVONE, Claudio, Una guerra civile - Saggio storico sulla moralità della Resistenza, Torino, Bollati Boringhieri, 1991.

35 DE FELICE, Franco, «Introduzione», in DE FELICE, Franco (a cura di), Antifascismi e Resistenze, Roma, La Nuova Italia Scientifica, 1997, pp. 11-39.

${ }^{36}$ LANARO, Silvio, Storia dell'Italia repubblicana: dalla fine della guerra agli anni novanta, Venezia, Marsilio, 1992, pp. 5-36. 
palcoscenici politici nazionali37. Il PCI esordì nel dopoguerra dovendo interpretare un ruolo sostanzialmente nuovo: nel giro di qualche mese e dopo alcuni decenni di precaria clandestinità, i comunisti dovettero inventarsi partito di massa: «all'indomani della caduta del regime fascista», ha scritto Angelo Ventrone, «il Partito comunista si trovò a dover costruire quasi dal nulla la propria legittimità agli occhi dell'opinione pubblica nazionale ed internazionale» ${ }^{8}$. Buona parte dell'apparato dirigente aveva passato in esilio gli anni del regime e quelli che potevano vantare una lunga militanza erano solo poche centinaia di uomini e di donne: «al piccolo manipolo di rivoluzionari professionali dei primi anni Quaranta si erano via via aggregate, sullo scorcio della guerra e nel corso della resistenza e dell'immediato dopoguerra, masse cospicue di nuovi militanti. Il crescendo era stato impetuoso: al momento del suo V congresso riunitosi a Roma il 29 dicembre 1945, il partito, secondo i dati ufficiali, poteva contare su 1.770.00o iscritti»39. Nel gennaio del 1948 Pietro Secchia avrebbe annunciato: «siamo ora un partito di massa»40. Passare da una ristretta cerchia di propagandisti e militanti ad un grande partito fu un processo veloce e la fase resistenziale ne rappresentò il primo decisivo passo. La Resistenza, e non i lunghi anni di militanza antifascista, ebbe quindi in sé tutte le caratteristiche per essere trasformata in momento mitico e venire "servita" al nuovo universo comunista in formazione, e così fu. Quella della guerra civile spagnola era invece un'esperienza troppo lontana e slegata dalle necessità di quel momento storico; o, quanto meno, non poteva aspirare ad avere quei presupposti epici che invece la Resistenza aveva. «Il mito della Resistenza», ha scritto Scoppola riferendosi non esclusivamente ai comunisti, «legato all'idea di un'Italia che ha subito il fascismo e che se ne è liberata per volontà e guerra di popolo è servito alla classe dirigente di fronte ai vincitori; è servito agli italiani sul piano psicologico; ma non risponde che per una parte limitata alla realtà». ${ }^{41} \mathrm{Fu}$ in questa fase che, a sinistra, l'immagine e la memoria dell'esperienza spagnola invece di conoscere un proprio sviluppo cominciò ad essere "appiattita” sulle vicende resistenziali e, com'era naturale che fosse, ci fu spazio solo per la brigata Garibaldi.

${ }^{37}$ Si veda: ELEY, Geoff, «Le eredità dell'antifascismo», in DE FELICE, Franco, op. cit., pp. 463468.

${ }^{8}$ VENTRONE, Angelo, La cittadinanza democratica - Come cattolici e comunisti hanno costruito la democrazia italiana, Bologna, Il Mulino, 2008, p. 32.

39 MICCOLI, Giovanni, «Cattolici e comunisti nel secondo dopoguerra: memoria storica, ideologia e lotta politica», in MICCOLI, Giovani, NEPPI MODONA, Guido, POMBENI, Paolo (a cura di), La grande cesura - La memoria della guerra e della resistenza nella vita europea del dopoguerra, Bologna, Il Mulino, 2001, p. 62.

40 NOVELLI, Enrico, Le elezioni del Quarantotto - Storia, strategie e immagini della prima campagna elettorale repubblicana, Roma, Donzelli, 2008, p. 14.

${ }^{41}$ SCOPPOLA, Pietro, op. cit., p. 12. 
Questo processo riguardò la storia dell'antifascismo nel suo complesso. Durante i primi anni del dopoguerra, con il nuovo assetto politico istituzionale ed in virtù delle necessità di cui sopra, si andò affermando un "discorso pubblico antifascista" che sarebbe stato una sorta di collante della memoria pubblica, in particolar modo tra le forze di sinistra, per gli anni a venire; allo stesso tempo, il conflitto che aveva animato la guerra civile (fascismo/antifascismo) «lungi da chiudersi con la caduta del regime, la fine della guerra e la fondazione della Repubblica» si sarebbe prolungato nel dopoguerra «come frattura non solo politica ma anche, addirittura, istituzionale» ${ }^{2}$. In virtù della ricerca di una narrazione il più possibile condivisa e che potesse andare oltre questo stato di conflitto persistente, oltre a perdere di vista la prospettiva internazionale dell'antifascismo a sinistra si tese ad esaurirlo nel momento resistenziale '43-'45 e a mettere in secondo piano le vicende riconducibili ai due decenni precedenti.43 Fu così che se da un lato l'antifascismo arrivò certamente ad essere «il fondamento stesso della Carta Costituzionale e lo strumento ideologico di legittimazione reciproca tra le forze politiche che in questa tradizione si riconoscevano» (in quel particolare momento il paradigma antifascista sembrò infatti essere l'unica forma storica in cui poteva essere incarnato e risolto il problema dell'unità nazionale dopo il crollo tanto del fascismo quanto della monarchia), dall'altro, se ne persero di vista la "memoria sul lungo periodo". ${ }^{4}$ Come ha scritto Alberto De Bernardi: «la riduzione dell'antifascismo ai suoi esiti resistenziali - in sostanza a premessa della Resistenza - decontestualizzandolo dal suo tempo ed allontanandolo dalla sua dimensione internazionale, rendeva inutilizzabili sul piano dell'elaborazione storiografica le chiavi di lettura essenziali per poter cogliere a pieno la sua identità e il suo significato nella storia novecentesca».45

${ }^{42}$ CHIARINI, Roberto, 25 aprile - La competizione politica sulla memoria, Venezia, Marsilio, 2005, pp. 13-14. Sulle memorie "altre" rispetto a quella antifascista nell'Italia del dopoguerra si vedano i due recenti studi: BALDASSINI, Claudia, L'ombra di Mussolini: l'Italia moderata e la memoria del fascismo, 1945-1960, Soveria Mannelli, Rubbettino, 2008 e LA ROVERE, Luca, L'eredità del fascismo: gli intellettualli, i giovani e la transizione al postfascismo, 1943-1948, Torino, Bollati Boringhieri, 2008.

43 Si veda a questo proposito: DE BERNARDI, Alberto, «Introduzione», in DE BERNARDI, Alberto; FERRARI, Paolo (a cura di), Antifascismo e identità europea, Roma, Carocci, 2004, pp. XI-XIII.

44 GALLERANO, Nicola, Le verità della storia - Scritti sull'uso pubblico del passato, Roma, Manifestolibri, 1999, p. 89.

45 DE BERNARDI, Alberto «Per una interpretazione dell'antifascismo: alcune ipotesi di indagine», in ALBARANI, Giuliano, OSTI GUERRAZZI, Amedeo, TAURASI, Giovanni (a cura di), Sotto il regime - Problemi, metodi e strumenti per lo studio dell'antifascismo,Milano, Unicopli, 2006, p. 49. 
Nei primi anni del dopoguerra si andò sviluppando quella che Filippo Focardi ha definito la narrazione egemonica della lotta antifascista ${ }^{46}$. Ci fu lo sforzo comune delle nuove forze politiche nel dipingere gli italiani come sostanzialmente un popolo di antifascisti, vittime, loro malgrado, del regime e della guerra di Mussolini ed in questo senso giocò un ruolo fondamentale il momento resistenziale presentato come riscossa di tutta una nazione; «già alla conclusione del conflitto l'antifascismo italiano delineò, dunque, la trama di una narrazione dell'esperienza della guerra e del fascismo che avrebbe costituito la base per la costruzione di una memoria collettiva largamente autoassolutoria»47. Ricollegandoci alla Spagna della guerra civile, fu proprio durante questi primi anni repubblicani che si assistette ad una rappresentazione posticcia dell'immagine delle Brigate Internazionali e dei volontari antifascisti: ogni riferimento a quell'esperienza sarebbe stato subordinato ad un rimando verso quella resistenziale, lo stesso ricorrente richiamo al "fronte populismo" che si sarebbe sperimentato in Spagna venne in realtà utilizzato strumentalmente in relazione al biennio 1943-1945; «la memoria della guerra di Spagna appare sin da subito indissolubilmente legata a quella della Resistenza»48. Con la radicalizzazione del conflitto politico in Italia, ne fu testimone la dura campagna per le elezioni del 1948, e l'entrata nel clima da guerra fredda le forze di sinistra tesero a chiudersi a riccio su una narrazione della Resistenza fortemente ideologizzata nella quale l'esperienza spagnola continuò a trovare una collocazione sbrigativa e semplicistica. In occasione delle celebrazioni per il 25 aprile 1948 Luigi Longo, figura di primo piano delle Brigate Internazionali, avrebbe parlato della Resistenza come di un movimento nazionale e popolare, «l'insurrezione di tutto un popolo» e si sarebbe rivolto all'Italia del «secondo Risorgimento»49. Questo riferimento alla storia italiana sul lungo periodo, che avrebbe conosciuto un certo successo all'interno della pubblicistica comunista nei primi decenni repubblicani, contribuì indirettamente alla semplificazione delle memorie spagnole; di quel volontariato rimase l'eco del rosselliano "Oggi in Spagna domani in Italia" (riproposto in maniera semplicistica), l'immagine del battaglione Garibaldi e poco altro. Il mondo cattolico e la DC, da parte loro, tanto per le violenze anticlericali che avevano contraddistinto la guerra civile (il Vaticano non aveva mai fatto mistero di riconoscersi nel bando franchista) quanto per la scarsissima presenza di propri rappresentanti tra

${ }^{46}$ Cfr FOCARDI, Filippo, La guerra della memoria - La resistenza nel dibattito politico italiano dal 1945 ad oggi, Roma-Bari, Laterza, 2005.

47 Ibidem, p. 10.

${ }^{48}$ FERUGLIO, Raffaele, «La memoria della guerra civile spagnola nella stampa del PCI 19481964», in Italia Contemporanea, 2007, n. 247, p. 272.

49 FOCARDI, Filippi, op. cit., pp. 23-24. Si veda anche BAIONI, Massimo, Risorgimento conteso - Memorie e usi pubblici nell'Italia contemporanea, Reggio Emilia, Diabasis, 2009, pp. 87-103. 
gli antifascisti in Spagna, non solo delegarono la memoria di quel volontariato alle forze di sinistra, ma anzi videro proprio in quello che era successo al clero iberico tra il 1936 ed il 1939 una delle legittimazioni del proprio feroce anticomunismo. Per i cattolici, sarebbe stato difficile, se non impossibile, riuscire ad inserire nella retorica resistenziale anche le vicende di chi aveva combattuto al lato dei repubblicani spagnoli5o. In estrema sintesi: da un lato si registrò la mitizzazione del momento resistenziale e dall'altro a questo venne de facto assimilata l'esperienza spagnola. Era quindi naturale che di quest'ultima si creassero delle memorie "frettolose" e "parziali", strettamente legate alle diverse fortune delle forze politiche di riferimento. Nella pratica, in virtù del protagonismo comunista e socialista e dalla sostanziale scomparsa del movimento anarchico e di quello azionista ci fu un eccessivo appiattimento sulla vicenda principale dell'antifascismo italiano in Spagna (il Battaglione Garibaldi), a scapito di qualsiasi altra esperienza (la Sezione Italiana).

Questa realtà ebbe dei riflessi anche sulla produzione storiografica. Nel 1982 Simona Colarizi, in apertura ad una raccolta di saggi dedicata all'emigrazione antifascista di area socialista, scrisse giustamente come l'attenzione che nel secondo dopoguerra gli storici avevano rivolto «soprattutto al periodo della Resistenza, considerata come il momento vitale dell'antifascismo» avesse relegato «i venti anni della lotta al fascismo in un limbo più o meno agiografico, dove la dura opposizione condotta durante tutto il periodo del regime nella clandestinità e nell'esilio aveva finito per essere valutata come una testimonianza, a volte eroica, di una minoranza che non aveva rinunciato ai propri ideali. [...] Nell'ottica della Resistenza, un bilancio dei vent'anni di milizia antifascista risulta estremamente ridimensionato, se non addirittura fallimentare nel suo obiettivo prioritario»; quello cioè di far cadere il regime mussoliniano ${ }^{51}$.

Facendo nostre queste considerazioni della storica italiana, possiamo vedere come in alcuni studi e ricerche "vicini" al mondo comunista le vicende dell'antifascismo in Spagna siano state ricostruite in maniera parziale e, a volte, strumentale. Paolo Spriano nella sua Storia del Partito Comunista Italiano, presentò l'epopea brigatista evitando sistematicamente qualsiasi menzione agli anarchici ed al loro contributo52. Sulla sua

5o A questo proposito vi veda: BOTTI, Alfonso, «Franco e i cattolici italiani», in Italia Contemporanea, 1996, n. 204.

${ }^{5}$ COLARIZI, Simona, «Problemi storiografici sul fuoruscitismo e sull'antifascismo socialista all'estero", in ARFÉ, Gaetano (a cura di), L'emigrazione socialista nella lotta contro il fascismo (1926-1939), Firenze, Sansoni,1982, p. 1.

$5^{2}$ SPRIANO, Paolo, Storia dela Partito comunista italiano - I fronti popolari, Stalin, la guerra, Torino, Einaudi, 1970, pp. 88-89. 
stessa lunghezza d'onda si pose anche Giorgio Amendola53. Roberto Battaglia, tralasciando le divisioni che ci furono e citando solo le Brigate Internazionali, parlò della guerra di Spagna come del «primo banco di prova dell'unità della Resistenza Italiana: comunisti, socialisti, gielle, repubblicani, anarchici», sarebbero stati, «fianco a fianco nella lotta comune», in una ricostruzione utilitaria al quadro interpretativo della guerra partigiana come secondo Risorgimento54. Luigi Longo, nelle suo Le Brigate Internazionali in Spagna dedicò ben poco spazio a chi non fece parte del Battaglione Garibaldi; «degli italiani tra i primi accorsi», scrisse, «ci sono Gorizia (Remigio Maurovich) ad Irun, Rosselli, Angeloni, Pellegrini, Scotti, Nino Nanetti sul fronte di Huesca, Fernando de Rosa sulla sierra; cioè comunisti, socialisti, repubblicani, seguaci di Giustizia e Libertà», nessun cenno meritarono invece i militanti libertari55.

Rimanendo sugli anarchici, tanto Sandro Attanasio quanto Giacomo Calandrone nei rispettivi volumi avrebbero entrambi parlato della Sezione Italiana come di una colonna giellista tralasciandone la natura libertaria56. Quello di appiattire il fronte antifascista costituito dai volontari stranieri sulla sola esperienza delle Brigate Internazionali senza così entrare nello specifico e nella complessità delle vicende spagnole fu quindi una tendenza che si affermò, all'interno della narrazione sull'antifascismo italiano, con successo. In particolare, tra i comunisti il ricorso a una narrazione simile fu anche uno straordinario strumento attraverso il quale veicolare un'educazione civile all'interno di una militanza che, come si è detto, era in "corso di formazione": «nei momenti di grande tensione politica, di disgregazione della compagine sociale», ha recentemente scritto Raffaele Feruglio riferendosi al PCI, «la memoria della guerra civile spagnola ha rappresentato un collante decisivo, uno strumento di auto identificazione nazionale e politica»57. Nel 1950, ad esempio, le celebrazioni per l'anniversario della fondazione delle BI si tennero a Parma, città simbolo della resistenza, alla presenza di Luigi Longo (PCI) e Pietro Nenni (PSI), due figure centrali dell'esperienza interbrigatista; in quell'occasione L'Unità volle mettere in relazione gli antifascisti del 1936 ed i protagonisti della guerra di Corea, che si stava combattendo in quei mesi58. Finalità "didattiche" del tutto simili a queste contraddistinsero anche la proliferazione, a partire dalla seconda metà degli anni

53 AMENDOLA, Giorgio, Storia del partito comunista italiano, 1921-1943, Roma, Editori Riuniti, 1978, pp. 303-305.

54 BATTAGLIA, Roberto, Storia della Resistenza Italiana, Roma, Editori Riuniti, 1953, p. 38.

55 LONGO, Luigi, Le Brigate Internazionali in Spagna, Roma, Editori Riuniti, 1972, p. 41.

${ }^{6}$ ATTANASIO, Sandro, Gli italiani e la guerra di Spagna, Milano, Mursia, 1974, pp. 63-71 e CALANDRONE, Giacomo, La Spagna brucia. Cronache garibaldine, Roma, Editori Riuniti, 1973 , p. 16.

57 FERUGLIO, Raffaele, op. cit., p. 271.

${ }^{8}$ Ibidem, p. 275. 
sessanta, delle autobiografie di chi aveva partecipato al conflitto spagnolo: la memorialistica venne interpretata come uno strumento attraverso il quale "trasmettere" alle generazioni più giovani i valori dell'antifascismo.59

Avviandoci alla conclusione, è utile citare un ultimo elemento che a nostro avviso ha influenzato nell'emersione di quelle dei comunisti e dei socialisti come le memorie forte del volontariato in Spagna: il riferimento a Giuseppe Garibaldi. L'immagine dell'eroe dei due mondi, il cui nome aveva contrassegnato la brigata a maggioranza italiana delle BI, sarebbe stato ripreso durante la Resistenza prima e nell'immediato dopoguerra poi. Le formazioni comuniste che si organizzarono durante gli ultimi mesi del 1943 vennero, infatti, intitolate proprio a Garibaldi con l'esplicita volontà, cui abbiamo già fatto riferimento, di legare la nuova lotta «al primo Risorgimento, a quel sentimento di schietto internazionalismo che fu tipico dei suoi uomini più avanzati, di Mazzini e di Garibaldi».60 Come ha scritto anche Mario Isnenghi, le bande partigiane raccolsero «ragazzi che sanno poco o nulla di politica» e a cui bisognava rivolgersi «con alfabeti semplici e chiari»: Garibaldi e il fazzoletto rosso furono fra questi simboli comunicativi e identitari. ${ }^{61}$ Garibaldi fu poi nuovamente ripreso in occasione della campagna elettorale per le elezioni del 18 aprile 1948, un voto che segnò «l'adeguamento dell'Italia al nuovo ordine geopolitico uscito dalla seconda guerra mondiale» e che fu il momento nel quale emerse la contrapposizione comunismo/anticomunismo ${ }^{62}$. «Garibaldi, uno dei padri dell'unità italiana», ha scritto Novelli a questo riguardo, «nel 1948 viene forzatamente arruolato in uno dei due schieramenti contrapposti, divenendo nel bene, ma soprattutto nel male, protagonista della campagna elettorale». 63

Questo nuovo protagonismo del nome e dell'immagine di Garibaldi negli anni che vanno dal 1943 al 1948 sicuramente contribuì a quel processo di appiattimento (e semplificazione) della figura del volontario antifascista su quella del "Garibaldino di Spagna”. Chi non aveva fatto parte delle BI o del battaglione Garibaldi si trovò sostanzialmente escluso da questa memoria e tese, inevitabilmente, a crearne una sua, alternativa. Questo fu il caso di chi combatté nella Sezione Italiana, la cui memoria, come si è già detto, rimase sostanzialmente delegata e relegata al movimento libertario: gli anarchici diedero vita ad una fitta memorialistica, che, a causa della marginalità del

59 Si veda: CASALI, Luciano, «La memoria ambigua - Guerra e rivoluzione in Catalogna negli scritti degli italiani», in Italia Contemporanea, 1987, n. 166), pp. 27-29.

6o BATTAGLIA, Roberto, op. cit., p. 347.

${ }^{61}$ ISNENGHI, Mario, Garibaldi fu ferito - Il mito, le favole, Roma, Donzelli, 2007, p. 143.

${ }^{62}$ NOVELLI, Enrico, op. cit., p. VIII.

63 Ibidem, p. 38. 
movimento libertario nell'Italia repubblicana, sarebbe rimasta sostanzialmente fuori dal dibattito pubblico. Enzo Traverso ha recentemente scritto come vi siano «memorie ufficiali, istituzionalizzate [....] e memorie sotterranee, nascoste [...] La visibilità ed il riconoscimento di una memoria dipendono anche dalla forza di coloro che la portano. In altre parole vi sono memorie forti e memorie deboli» 64 . Non v'è dubbio che quella dei libertari, nell'Italia del dopoguerra, sia stata una memoria debole, una "memoria altra" rispetto a quella di chi aveva fatto parte delle Brigate Internazionali ed era stato "garibaldino".

\section{Conclusioni}

Come si è visto, durante il dopoguerra ai presupposti cui abbiamo fatto riferimento nella prima parte di questo saggio fece effettivamente seguito una pluralità di memorie; nelle ultime pagine si poi è cercato d'inserire le vicende delle memorie spagnole nel contesto mobile e complicato dell'Italia del dopoguerra. L'elemento saliente che ci pare essere emerso dalla nostra analisi è l'appiattimento di una parte di quelle memorie (soprattutto le comuniste) sulle vicende resistenziali ed il contemporaneo determinarsi, in opposizione a questo processo, di memorie deboli e residuali (quelle libertarie). Crediamo sia quindi giusto utilizzare tanto il plurale quanto l'aggettivo difficili nel riferirsi alle memorie del volontariato italiano in Spagna.

64 TRAVERSO, Enzo, Il passato: istruzioni per l'uso. Storia, memoria, politica, Verona, Ombre Corte, 2006, p. 51. 
Vorremmo chiudere con un riferimento ad una questione più generale. Ha senso oggi, come storici, tornare ad interrogarsi su temi tanto praticati come quelli dell'antifascismo e della sua memoria? In Italia, dai primi anni '90, si è andato affermando, come l'ha brillantemente definito Sergio Luzzatto, il verbo postantifascista di cui una delle priorità è stata la supposta necessità di una memoria condivisa in nome della quale si sono messe sul banco degli imputati le stesse radici antifasciste della Repubblica Italiana ${ }^{65}$. Qualche anno fa Marcello Pera, all'epoca Presidente del Senato, si spinse sino a parlare dell'antifascismo come di un «mito incapacitante» del nostro ordinamento ${ }^{66}$. In realtà, l'antifascismo e la sua memoria, pur richiedendo di profonde revisioni che ne ridefiniscano i confini spazio-temporali, possono aspirare ad avere ancora una loro attualità; recentemente Alberto de Bernardi ha scritto come la tradizione antifascista rappresenti «oggi più di ieri, un campo di questioni aperte e di interrogativi irrisolti, piuttosto che un solido edificio di certezze e di risposte convincenti» ${ }^{67}$. Partire dalla difficile memoria dell'esperienza spagnola, per quanto riguarda lo specifico di questo nostro studio, potrebbe essere un interessante spunto per interrogarsi sulla lunga durata del movimento antifascista e per analizzarne i momenti conflittuali. In sintesi, la pluralità di memorie generate dal volontariato in Spagna può forse essere interpretata come la testimonianza delle interessanti prospettive di ricerca che si presentano allo studioso intenzionato a confrontarsi con questo tema di ricerca.

${ }^{65}$ LUZZATTO, Sergio, La crisi dell'antifascismo, Torino, Einaudi, 2004, pp. 10-13 e 23.

${ }^{66}$ Ibidem, p. 33.

${ }_{67}$ DE BERNARDI, Alberto, op. cit., p. XIV. 


\section{* L'autore}

Enrico Acciai: si è addottorato in storia dell'Europa contemporanea presso l'Università degli Studi della Tuscia, nel corso del 2010 ha svolto attività di ricerca grazie ad una borsa della Fondazione Luigi Salvatorelli. Già titolare di un assegno di ricerca presso il dipartimento per lo Studio delle Lingue e delle Civiltà Classiche e Moderne (CICLAMO) dell'Università degli Studi della Tuscia è attualmente ricercatore presso il departamento de Historia Moderna y Contemporanea della Universidad de Cantabria. Nel 2011 è inoltre risultato vincitore del premio annuale bandito dall'Associazione Casa Di Vittorio. Si è occupato di storia dell'anarchismo spagnolo e dell'antifascismo italiano; attualmente sta portando avanti un progetto di ricerca sui volontari italiani che presero parte alla guerra civile.

URL: < http://studistorici.com/progett/autori/\#Acciai >

\section{Per citare questo articolo:}

ACCIAI, Enrico, «Memorie difficili. Antifascismo italiano, volontariato internazionale e guerra civile spagnola», Diacronie. Studi di Storia Contemporanea: Spagna Anno Zero: la guerra come soluzione, 29/07/2011, URL:< http://www.studistorici.com/2011/07/29/acciai1_numero_7/>

Diacronie Studi di Storia Contemporanea $\mathcal{B}$ www.diacronie.it

Risorsa digitale indipendente a carattere storiografico. Uscita trimestrale. redazione.diacronie@hotmail.it

Comitato di redazione: Marco Abram - Giampaolo Amodei - Jacopo Bassi - Luca Bufarale - Alessandro Cattunar - Alice De Rensis Barbara Galimberti - Deborah Paci - Fausto Pietrancosta - Martina Sanna - Matteo Tomasoni - Luca Zuccolo

Diritti: gli articoli di Diacronie. Studi di Storia Contemporanea sono pubblicati sotto licenza Creative Commons 2.5 Possono essere riprodotti a patto di non modificarne i contenuti e di non usarli per fini commerciali. La citazione di estratti è comunque sempre autorizzata, nei limiti previsti dalla legge. 